\title{
ENFANT, J'AIMAIS LES ÉPINARDS : L'EXPRESSION NOMINALE DU TEMPS
}

\author{
Angelina Aleksandrova
}

Armand Colin | « Langages »

2012/4 n 188 | pages 95 à 110

ISSN 0458-726X

ISBN 9782200927776

Article disponible en ligne à l'adresse :

http://www.cairn.info/revue-langages-2012-4-page-95.htm

\section{Pour citer cet article :}

Angelina Aleksandrova, «Enfant, j'aimais les épinards : l'expression nominale du temps », Langages 2012/4 ( $\left.\mathrm{n}^{\circ} 188\right)$, p. 95-110.

DOI 10.3917/lang.188.0095

Distribution électronique Cairn.info pour Armand Colin.

(C) Armand Colin. Tous droits réservés pour tous pays.

La reproduction ou représentation de cet article, notamment par photocopie, n'est autorisée que dans les limites des conditions générales d'utilisation du site ou, le cas échéant, des conditions générales de la licence souscrite par votre établissement. Toute autre reproduction ou représentation, en tout ou partie, sous quelque forme et de quelque manière que ce soit, est interdite sauf accord préalable et écrit de l'éditeur, en dehors des cas prévus par la législation en vigueur en France. Il est précisé que son stockage dans une base de données est également interdit. 


\section{Enfant, j'aimais les épinards : l'expression nominale du temps}

\section{INTRODUCTION ${ }^{1}$}

Cette étude propose de revenir sur les relations entre une structure syntaxique bien connue en français - la construction détachée $\left(C D^{2}\right)$ - et le(s) sens qui lui est (sont) habituellement associé(s). En nous appuyant sur l'analyse de quelques noms d'humains $(\mathrm{NH})$ qui dénotent un être humain pendant les différentes phases de sa vie ${ }^{3}$ (p. ex. bébé, enfant, adolescent, adulte, vieillard, appelés des noms d'âge, $\mathrm{N}$-[âge]), nous reviendrons sur l'interprétation des $\mathrm{CD}$ nominales et, plus précisément, sur la possibilité de leur attribuer une valeur temporelle. Le travail sera enrichi par un aspect contrastif qui prendra deux dimensions : une comparaison avec d'autres $\mathrm{NH}$ (p. ex. les $\mathrm{N}$ de professions) et l'examen de la situation en bulgare pour la même structure en vue d'une meilleure compréhension de la spécificité française. Le but est non seulement de montrer que les $\mathrm{CD}$ nominales à valeur temporelle ne sont pas aussi rares que la littérature sur la question ne le laisse entendre, mais aussi d'attirer l'attention sur les relations entre le domaine nominal et l'expression du temps. De façon plus générale, notre étude vise à alimenter la réflexion sur la variabilité linguistique, et cela au moins à deux niveaux d'analyse : d'une part, en nous focalisant sur

1. Nous tenons à remercier Catherine Schnedecker pour ses conseils avisés, Dejan Stosic pour ses relectures attentives et constructives, et nos évaluateurs anonymes pour leurs remarques instructives.

2. Les CD ont été le sujet de nombreux travaux, entre autres : Apothéloz, Combettes \& Neveu (2009) ; Combettes (1998, 2005) ; Flaux \& Stosic (éds) (2007) ; Forsgren (2005) ; Forsgren, Jonasson \& Kronning (éds) (1998) ; Havu (2002a).

3. Il est important de noter que nous nous sommes intéressée à ces noms uniquement en tant que signifiant l'âge (enfant notamment est un terme polysémique et peut dénoter un individu du point de vue de sa filiation). 
le français, il s'agit de voir dans quelle mesure l'expression du temps peut prendre des «formes », sinon inattendues, du moins " peu attendues » avec les $\mathrm{NH}$; d'autre part, il s'agit d'examiner la variabilité inter-langues pour une même structure. Les analyses et les conclusions qui en découlent sont basées sur un corpus constitué d'occurrences extraites de Frantext (textes essentiellement littéraires pour la période 1970-2006) et de Wortschatz ${ }^{4}$ (articles de presse). Pour le bulgare, les occurrences sont extraites à la fois de Wortschatz, du Corpus National Bulgare (maintenu par l'Académie des Sciences de Sofia) et du Corpus Braun (cf. bibliographie). Nous avons travaillé sur un total de 126 occurrences ${ }^{5}$ de $\mathrm{CD}(\mathrm{N}$-[âge]) en français, leur traitement s'est fait à l'aide du concordancier AntConc et du logiciel de gestion de base de données Access.

L'article sera organisé en deux parties consacrées respectivement au réexamen des critères définitoires des $\mathrm{CD}$ temporelles $(\mathrm{CDtmp})^{6}$ et à l'étude comparative avec le bulgare.

\section{INTERPRÉTATION TEMPORELLE DES CD NOMINALES}

Les critères signalés comme obligatoires par B. Combettes (1998a), permettant de tracer la frontière entre les $C D$ et $\mathrm{d}$ 'autres constructions " périphériques " (adverbes de phrase, circonstants, etc.) sont au nombre de quatre : critère de détermination, de position ${ }^{7}$, de prédication seconde et de co-référence. Deux catégories de $C D$ sont usuellement distinguées : les CD qui prennent la valeur $\mathrm{d}^{\prime}$ une circonstancielle et celles qui ont un rôle descriptif. Traditionnellement, l'attribution d'une valeur aux CD va de pair avec l'identité morphosyntaxique de ses constituants. C'est ainsi que la parenté avec le domaine verbal mène B. Combettes à postuler que « les $\mathrm{CD}$ temporelles sont essentiellement représentées par les constructions participiales et par les gérondifs » (1998a : 48) et explique que les études sur l'interprétation temporelle des CD se sont penchées notamment sur ces formes (cf. Amourette 2004 ; Blanche-Benveniste 1998 ; Borillo 2006a, 2009). Pourtant, il a été observé de manière disparate que les N-[âge] peuvent recevoir une interprétation temporelle. J.-C. Anscombre parle d'une « construction curieuse, apparemment limitée à certains substantifs, lesquels caractérisent ou peuvent caractériser une période temporelle » (1990:287) ; L. Picabia dit ne connaître qu'une classe de $\mathrm{N}$ « pouvant s'interpréter naturellement comme des ancrages temporels » $(2000: 74)^{8}$ et C. Amourette remarque que la valeur

4. Étant donné qu'il s'agit d'une base de données alimentée régulièrement, précisons que le corpus a été constitué fin 2008, date de début de notre travail de thèse.

5. Il s'agit du nombre d'occurrences à valeur temporelle sur un total de 133 occurrences de CD(N-[âge]).

6. L'espace accordé ne nous permet pas de revenir en détail sur les critères définitoires des CD en général, ni sur l'opposition CD temporelles/CD qualitatives (cf. Havu 2002 ; Flaux \& Stosic (éds) 2007).

7. Cf. sur ce point Havu \& Pierrard (2007).

8. L'exemple de Picabia est le suivant : Enfant, Marie faisait des cauchemars. 
temporelle provient du sémantisme du lexème ${ }^{9}$ qui doit dénoter une période temporelle «caractéristique de l'existence du sujet » (2009: 45). Le point commun à ces observations est qu'elles concernent une tournure particulière - les CD.

\subsection{Retour sur les critères}

Sur les 133 occurrences de $\mathrm{CD}\left(\mathrm{N}\right.$-[âge]) examinées, $126^{10}$ (presque $95 \%$ ) se voient attribuer une interprétation temporelle, ce qui vient à l'encontre de la lecture "descriptive » associée aux $\mathrm{N}$, et demandent un regard plus attentif. Étant donné que les $\mathrm{N}$-[âge] entretiennent un rapport particulier avec le temps (Aleksandrova 2010), nous allons comparer leur fonctionnement avec d'autres $\mathrm{NH}$, comme les $\mathrm{N}$ de professions (appelés encore $\mathrm{N}$ de statut (Swart, Winter \& Zwarts 2007) ou N de rôle (Fauconnier 1984 ; Riegel 1985)).

\subsubsection{Détermination}

Considérons les énoncés suivants : (1) comporte un $\mathrm{N}$ de profession et (2) un $\mathrm{N}$-[âge] :

(1) Architecte, il travaillait beaucoup.

(2) Enfant, il n'aimait pas les épinards.

Dans les deux exemples, nous avons affaire à un prédicat en $C D$ initiale, en coréférence avec le sujet de la prédication principale (il) et constituant une prédication seconde. Nous allons y revenir, mais remarquons de suite que les deux $\mathrm{CD}$ sont sémantiquement équivalentes à une subordonnée temporelle en quand et qu'elles n'admettent pas une détermination (du moins dans cette position) :

(3) Quand il était architecte, il travaillait beaucoup.

(4) Quand il était enfant, il n'aimait pas les épinards.

(5) *Un/L'architecte, il travaillait beaucoup.

(6) *Un/L'enfant, il n'aimait pas les épinards.

En réalité, l'absence d'article est un critère nécessaire, mais non suffisant, pour une lecture temporelle. Dans l'exemple suivant, où le prédicat d'âge est postposé au sujet, l'absence d'article indéfini n'entraîne pas une interprétation temporelle, et le SP de deux ans impose la lecture qualitative :

(7) Albertine, Norbert et, devant eux sur un support très artificiel comme l'est toujours le mobilier chez un photographe, sur une espèce de guéridon très haut - Charlotte, enfant de deux ans, portant un bonnet orné de dentelles et une robe de poupée. (Makine, Le Testament français, 1995)

$\rightarrow{ }^{*}$ Charlotte, quand elle était enfant de deux ans, portant un Bonnet.

9. Les exemples de Amourette sont: Jeune, il avait la tête étroite et Enfant, il buvait du café au lait.

10. Cf. Tableau 1 . 
Suite aux observations faites jusqu'ici, on peut dire que, hormis la contrainte imposée par le sémantisme propre de chaque type de $\mathrm{N}$-[âge] ${ }^{11}$, deux facteurs sont nécessaires à son interprétation temporelle en CD : l'emploi «nu » (sans déterminant, ni modifieur) et la position frontale. Arrêtons-nous un peu plus sur ce dernier point.

\subsubsection{Position}

Concernant la corrélation entre la position initiale et la valeur temporelle que l'on assigne à la $\mathrm{CD}$, notre propos doit être pondéré. Les $\mathrm{N}$-[âge] peuvent recevoir une valeur temporelle en $\mathrm{CD}$ ailleurs dans la proposition. En témoignent les exemples suivants :

(8) René, quant à lui, adolescent, a vu les bombes tomber sur Vaise. (Le Parisien, désormais LP)

(9) J'ai émigré, enfant, vers la France, avec ma mère, après la deuxième guerre mondiale pour fuir l'armée rouge et le communisme. (LP)

(10) J'ai grandi ailleurs, loin de ce qui m'entourait, me retenait, par force, enfant. (Bianciotti, Le pas si lent de l'amour, 1995)

Le tableau 1 présente les occurrences de CDtmp(N-[âge]) recensées et leur répartition a) en fonction de la position dans laquelle elles apparaissent dans la proposition ${ }^{12}$ (en position initiale, intégrée ou finale) et $b$ ) en fonction du type de phrase où elles apparaissent. Pour les besoins de l'analyse, nous avons distingué les modes de construction des phrases complexes (notamment en indiquant si la CD apparaît dans une proposition coordonnée, une matrice ou une subordonnée) :

Tableau 1 : Position CDtmp (N-âge)

\begin{tabular}{|c|c|c|c|c|c|c|c|c|c|c|c|c|c|}
\hline & \multirow{3}{*}{ 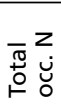 } & \multicolumn{4}{|c|}{ Position Initiale } & \multicolumn{4}{|c|}{ Position Intégrée } & \multicolumn{4}{|c|}{\begin{tabular}{|l|} 
Position Finale \\
\end{tabular}} \\
\hline & & \multirow{2}{*}{ P simple } & \multicolumn{3}{|c|}{ P complexe } & \multirow{2}{*}{ P simple } & \multicolumn{3}{|c|}{$\mathrm{P}$ complexe } & \multirow{2}{*}{ P simple } & \multicolumn{3}{|c|}{ P complexe } \\
\hline & & & Co. & Mat. & Sub. & & Co. & Mat. & Sub. & & Co. & Mat. & Sub. \\
\hline Enfant & 78 & 6 & 8 & 11 & 12 & 10 & 4 & 2 & 12 & 1 & & 7 & 5 \\
\hline Adol. & 37 & 9 & 2 & 2 & 12 & 6 & 1 & & 5 & & & & \\
\hline Adulte & 11 & 2 & 3 & & 3 & 2 & 1 & & & & & & \\
\hline \multirow[t]{2}{*}{ TOTAL } & 126 & 17 & 13 & 13 & 27 & 18 & 6 & 2 & 17 & 1 & 0 & 7 & 5 \\
\hline & & \multicolumn{4}{|c|}{70 occ. $(55,5 \%)$} & \multicolumn{4}{|c|}{43 occ. $(34,1 \%)$} & \multicolumn{4}{|c|}{13 occ. $(10,3 \%)$} \\
\hline
\end{tabular}

D'emblée on remarque que les CDtmp apparaissent de façon privilégiée en position initiale ou en incise dans une proposition ; la position finale, en revanche, accueille beaucoup plus difficilement les CDtmp. Le tableau appelle au moins deux remarques. D'abord, enfant apparaît de façon plus prononcée en CD contrairement à adolescent ou adulte, ce qui peut être expliqué par le fait que enfant est

11. Une étude approfondie sur une palette plus large de NH devra apporter plus de lumière sur les contraintes d'ordre sémantique. Ce travail sort du cadre que nous nous sommes donné ici. Les spécificités des $\mathrm{N}$-[âge] sont étudiées dans la section suivante.

12. Pour une meilleure lisibilité, nous notons la position initiale (1), la position intégrée (2) et la position en fin de proposition (3). 
le N-[âge] quantitativement le plus présent dans notre corpus global ${ }^{13}$. Ensuite, il faut noter l'absence de CD avec bébé ou vieillard, mais, de toute évidence, cela est une particularité de notre corpus, parce qu'il est tout à fait envisageable de concevoir un énoncé comme Bébé, tu étais adorable, vieillard, tu seras pénible.

\subsubsection{Co-référence}

En tenant compte des travaux de D. Wiggins (2001) et de la problématique (au départ philosophique) de l'identité à travers le temps, dans un exemple comme Enfant, j'aimais les épinards, il nous paraît plus plausible de parler de " coréférence partielle ». En effet, si le lien coréférentiel avec le sujet de la principale est indéniable ${ }^{14}$ - enfant dénote une phase obligatoire de l'existence d'un être humain - ici la coréférence est une coréférence d'identité numérique (il s'agit toujours d'un seul et unique individu, une " entité sortale » dans les termes de D. Wiggins), mais non une coréférence de temps (cf. infra). De même, quand il s'agit d'une CDtmp (N profession) - comme dans Architecte, il travaillait beaucoup - il y a coréférence de l'individu, mais non coréférence de rôle, si le sujet n'est plus architecte au moment de l'énonciation.

\subsection{Les CDtmp(N-[âge]) : des circonstants ?}

Si la coréférence et la prédication seconde ${ }^{15}$ permettent de distinguer dans une certaine mesure les $\mathrm{CD}$ des circonstants, les $\mathrm{CDtmp}$ (N-[âge]) ont un comportement similaire avec ces derniers.

\subsubsection{Subordonnée sous-jacente}

Reprenons les deux énoncés du début. Il convient de remarquer, d'abord, que, contrairement à (1), (2) n'équivaut ( $\rightarrow$ ) ni à une principale (13) ni à une relative restrictive (14) - remarque de $C$. Amourette (2009) -, tandis que l'on peut très bien concevoir (11) et (12) à partir de (1) :

(11) Il était architecte, il travaillait beaucoup.

(12) Lui, qui était architecte, il travaillait beaucoup.

(13) $\nrightarrow$ Il était enfant, il n'aimait pas les épinards.

(14) $\nrightarrow$ Lui, qui était enfant, il n'aimait pas les épinards.

Seul l'exemple avec le $\mathrm{N}$-[âge] permet la récupération de la subordonnée temporelle sans poser de problème. Contrairement au contexte neutre de l'exemple construit (1), les CD avec un Npro donnent lieu à une telle paraphrase beaucoup plus facilement dans un contexte contrastif du type Architecte, il travaillait beaucoup, mais depuis sa retraite il ne fait plus rien. L'interprétation temporelle des Npro,

13. C'est-à-dire l'ensemble des données où nous avons cherché les N-[âge] en CD (cf. Introduction).

14. Il nous semble que dans la mesure où différents entités linguistiques renvoient au même référent, on peut parler de coréférence entre X-enfant, X-adolescent, etc. avec la précision que la « distance temporelle » entre chaque phase fait que nous préférons l'étiquette de «coréférence partielle ».

15. Définie par Combettes comme « une sorte de proposition réduite qui, après ellipse du sujet, ne conserverait que le prédicat ou qu'une partie du prédicat » (1998a : 12). 
plus que pour les $\mathrm{N}$-[âge], est fortement contrainte par le contexte, même si elle ne semble pas exclue dans (1). En tout cas, la lecture temporelle d'une $\mathrm{CD}(\mathrm{Npro})$, paraît plus évidente d'un côté, quand la prédication principale est au futur :

$$
\text { Professeur, tu seras riche. } \rightarrow \text { Quand tu seras professeur, tu seras riche. }
$$

$$
\text { Directeur, il aura les bras longs. } \rightarrow \text { Une fois directeur, il aura les bras longs. }{ }^{16}
$$

et de l'autre côté, elle semble facilitée par l'emploi de la copule (comme dans (1), plutôt qu'avec un autre verbe : Architecte, il était riche $(? \rightarrow$ Quand il était architecte, il était riche).

\subsubsection{Mobilité}

Postuler que la CDtmp peut être assimilée à une subordonnée circonstancielle, et/ou jouer le rôle d'un circonstant de temps demande d'analyser sa capacité à occuper différentes positions dans la proposition. Même si, par ailleurs, il peut y avoir des compléments de temps peu mobiles, il s'agit de vérifier l'hypothèse initiale selon laquelle une CDtmp doit jouir d'une plus grande liberté par rapport à une CDqua, qui entretient des liens plus forts avec l'élément qu'elle qualifie dans la prédication principale.

D'après l'analyse de notre corpus, les CDtmp(N-[âge]) apparaissent de façon récurrente dans des phrases complexes : 90 occurrences vs 36 occurrences dans des phrases simples. De manière systématique, nous avons testé les possibilités de déplacement pour chaque occurrence en envisageant quatre cas de figure : de sa position dans l'énoncé vers, respectivement, les deux autres positions possibles ; déplacement à la fois vers les deux autres positions possibles (p. ex. $1>2 / 3)$; enfin impossibilité de déplacement. Deux classements sont proposés : en fonction du type de la phrase et en fonction du N-[âge] correspondant.

\begin{tabular}{|c|c|c|c|c|c|c|c|c|c|c|c|c|}
\hline & \multicolumn{4}{|c|}{ Position initiale (1) } & \multicolumn{4}{|c|}{ Position integrée (2) } & \multicolumn{4}{|c|}{ Position finale (3) } \\
\hline & $1>2$ & $1>3$ & $1>2 / 3$ & imp. & $2>1$ & $2>3$ & $2>1 / 3$ & imp. & $3>1$ & $3>2$ & $3>1 / 2$ & imp \\
\hline Phrase simple & 3 & 5 & 1 & 8 & 9 & 1 & 2 & 6 & & & 1 & \\
\hline Prop coordonnée & 3 & 3 & 2 & 5 & 4 & 1 & 1 & & & & & \\
\hline Matrice & 6 & 4 & 1 & 2 & 3 & & & & 5 & & 1 & 1 \\
\hline Subordonnée & 15 & 3 & 4 & 6 & 5 & 2 & 5 & 3 & 3 & 1 & & 1 \\
\hline
\end{tabular}

Tableau 2. Mobilité au sein de la proposition

\begin{tabular}{|l|c|c|c|c|c|c|c|c|c|c|c|c|}
\cline { 2 - 12 } \multicolumn{1}{c|}{} & \multicolumn{4}{c|}{ Position initiale (1) } & \multicolumn{5}{c|}{ Position integrée (2) } & \multicolumn{5}{c|}{ Position finale (3) } \\
\cline { 2 - 12 } \multicolumn{1}{c|}{} & $1>2$ & $1>3$ & $1>2 / 3$ & imp. & $2>1$ & $2>3$ & $2>1 / 3$ & imp. & $3>1$ & $3>2$ & $3>1 / 2$ & imp. \\
\hline enfant & 12 & 9 & 7 & 9 & 15 & 3 & 5 & 5 & 8 & 1 & 2 & 2 \\
adolescent & 14 & 4 & 1 & 7 & 3 & 1 & 3 & 4 & & & & \\
adulte & 1 & 2 & & 5 & 3 & & & & & & & \\
\hline
\end{tabular}

Soulignons les points les plus marquants qui ressortent de ces deux tableaux :

16. L'espace ne nous permet pas de développer plus en avant la notion de phase, mais notons qu'en fonction de la phase de vie sélectionné, l'introducteur de la subordonnée change : avec adulte, on sélectionne plutôt la borne initiale (Une fois adulte, Quand je suis devenu adulte vs. *Quand j'étais adulte). Par ailleurs, d'autres variantes doivent aussi être examinées dans le futur (alors que, lorsque, tant que, en tant que). 
- la CDtmp(N-[âge]) occupe de façon privilégiée les positions 1 et 2, et cela indépendamment du type de phrase ou du N-[âge] ${ }^{17}$. De même, le déplacement entre ces deux positions est plus aisé que les variantes $2>3,1>3,3>1,3>2$;

- un fait qui doit être expliqué est l'impossibilité de déplacement, relativement importante à la fois pour les CDtmp en position 1 et 2 ;

- même si enfant est le N-[âge] quantitativement le plus présent dans le corpus, on remarquera que c'est le seul qui apparaît en 3 (le plus souvent en fin de matrice, i.e. juste avant la conjonction de subordination).

En fait, les contraintes qui pèsent sur la mobilité de la CDtmp(N-[âge]) sont multiples et d'ordre différent. Sur le plan syntaxique, il s'agit de déterminer la distribution sur la chaîne syntagmatique et les dépendances existantes. À un niveau pragmatico-discursif, entrent en jeu des facteurs de cohérence textuelle (notamment l'établissement de la coréférence avec l'élément de la principale). Enfin, il faut tenir compte des spécificités sémantiques de chaque nom, ainsi que des contraintes d'ordre plutôt stratégique, d'organisation et progression discursive, qui peuvent se greffer au reste. Contentons-nous de signaler trois faits, remarqués par ailleurs (voir par exemple Fuchs \& Fournier 2003), auxquels les CDtmp(N-[âge]) n'échappent pas.

Mobilité inter-propositionnelle. Globalement, dans les phrases complexes, la $\mathrm{CDtmp}(\mathrm{N}$-[âge]) est contrainte de rester dans les limites de la proposition où elle apparait, surtout quand il s'agit de deux sujets différents :

(17) Les Français écoutent un Palestinien raconter comment, enfant, il a vu sa famille massacrée par les phalanges chrétiennes avec le consentement de l'armée israélienne. (2002-04-20.LM)

$\rightarrow{ }^{*}$ Enfant, les Français écoutent un Palestinien raconter comment il a vu sa famille massacrée...

(18) Mohammed Atta était un garçon solitaire qui, adolescent, ne se joignait pas à ses camarades allant draguer des filles à la sortie du lycée. (2002-09-10.LM) $\rightarrow$ Adolescent, Mohammed Atta était un garçon solitaire qui ne se joignait pas à ses camarades...

Les contraintes sur la mobilité inter-propositionnelle sont, la plupart du temps, liées à l'étendue de la portée des $\mathrm{CD}$ et ces faits seront examinés plus en détail au $\S 2.2 .3$.

Mobilité intra-propositionnelle. Même si la $\mathrm{CD}$ a nécessairement un caractère extra-prédicatif ${ }^{18}$, le GV peut se révéler un obstacle à sa mobilité. C'est notamment le cas quand le GV est plus élaboré. Si le déplacement de la CD en position thématique ne pose pas de problèmes, elle a du mal à apparaître en position 3 , après trois compléments de lieu qui vont la séparer du COD coréférant :

17. Sur le circonstants en position initiale voir notamment Le Querler (1993).

18. Autrement dit, elle n'est ni régie ni appelée par le verbe de la principale. 
La carrière diplomatique de son père l'a conduit, enfant, en Côte d'Ivoire, à Djibouti, au Burundi. (Le Monde, LM)

La carrière diplomatique de son père l'a conduit en Côte d'Ivoire, à Djibouti, au Burundi, *enfant.

Les liens sémantico-référentiels entre la $C D$ et un élément de la principale (le sujet en l'occurrence), font qu'elle ne jouit pas de la liberté d'un circonstant. Il se trouve que, parfois, l'établissement de la coréférence peut être, lui-même, un facteur contraignant la mobilité de la CDtmp(N-[âge]), surtout si le COD renvoie à un référent [+humain] comme dans :

(21) Entre-temps, il y a eu la pension où, adolescent $\mathrm{i}_{\mathrm{i}} \mathrm{il}_{\mathrm{i}}$ rencontre le peintre Gérard Garouste. (LM)

$\rightarrow$ il y a eu la pension, où il rencontre le peintre Gérard Garouste, adolescent.

\subsubsection{Portée ${ }^{19}$}

La position initiale fait l'unanimité parmi les linguistes pour être considérée comme faisant partie de la zone thématique. Il s'agit d'un espace qui accueille de façon privilégiée les éléments d'un faible apport informationnel, constituant le thème de l'énoncé ${ }^{20}$, ce qui est, ou présupposé être, connu. On comprend pourquoi les circonstants spatio-temporels occupent aisément cette zone initiale ils instaurent ainsi le cadre dans lequel le reste de la proposition a lieu, en jouant ainsi le rôle d'un repère.

Dans nos données, nous avons observé, d'une part, que la majorité (55.56 \%) des CDtmp (N-[âge]) occupent la position 1 et, d'autre part, que la mobilité vers celle-ci est beaucoup plus aisée (dans $69,6 \%$ des cas de positions 2 et 3). Cidessous, les CDtmp(N-[âge]) enfant, adolescent et adulte se trouvent en position initiale (adulte étant à l'initiale d'une proposition coordonnée) :

(22) Enfant, là où j'allais en vacances, je lisais la pancarte. (Dolto, La cause des enfants, 1985)

(23) Adolescent, Michel Chassang se rêvait notable ; adulte, il regrette d'être pris pour un "technicien". (2002-06-19. LM)

À la manière d'un circonstant temporel, les CD forment un «cadrage extérieur » (Fuchs \& Fournier, 2003 : 95). Ce caractère extraprédicatif et thématique se confirme par des tests bien connus (négation, interrogation). En somme, nous ne pouvons que confirmer ces faits, sans noter de fonctionnement particulier aux CDtmp(N-[âge]) par rapport à n'importe quel circonstant : la portée s'étend à la totalité de la proposition avec les effets de sens attendus traditionnellement associés aux positions ("cadratif » ou " scénique » pour la position 1, effet de « re-cadrage » ou « cadrage à l'envers » (ibid.) pour la position 3).

19. Sous portée, nous entendons l'étendue de l'effet produit par le complément circonstanciel.

20. Dans le cadre de cette étude, nous allons adopter la distinction thème-rhème. Nous sommes consciente de la complexité de cette distinction qui, comme c'est souvent le cas avec certaines notions en linguistique, souffre d'une surexploitation. 
Nous avons déjà pu observer que la mobilité inter-propositionnelle de la $\mathrm{CDtmp}(\mathrm{N}$-[âge]) entraîne obligatoirement un changement de portée. Considérons les exemples suivants, où la $\mathrm{CD}$ se trouve immédiatement après le subordonnant :

(24) Mohammed Atta était un garçon solitaire qui, adolescent, ne se joignait pas à ses camarades allant draguer des filles à la sortie du lycée. (LM) Quand on vit dans l'anticipation d'une rupture - parce que, enfant, on en a vécu une traumatisante -, on peut, de façon inconsciente, aller jusqu'à la provoquer. (LP) Garouste. (LM)}

Entre-temps, il y a eu la pension où, adolescent, il rencontre le peintre Gérard

Dans tous les exemples, la CD porte sur la proposition enchâssée. Cependant, les possibilités de déplacement ne sont pas les mêmes. Au sein de la subordonnée, la CD peut prendre la position finale seulement dans (28), ce qui est exclu pour des raisons de longueur du GV en (27) et d'ambiguïté co-référentielle dans (29) ${ }^{21}$ :

$$
\begin{aligned}
& \text { *Mohammed Atta était un garçon solitaire qui ne se joignait pas à ses } \\
& \text { camarades allant draguer des filles à la sortie du lycée, adolescent. } \\
& \text { Quand on vit dans l'anticipation d'une rupture - parce qu'on en a vécu } \\
& \text { une traumatisante, enfant -, on peut, de façon inconsciente, aller jusqu'à la } \\
& \text { provoquer. } \\
& \text { ?Entre-temps, il y a eu la pension, où il rencontre le peintre Gérard Garouste, } \\
& \text { adolescent. }
\end{aligned}
$$

Si la CD franchit les limites de la proposition subordonnée d'accueil, on se heurte à d'autres contraintes. Envisageons deux cas de figures : le déplacement de la $\mathrm{CD}$ en position initiale et en position finale de matrice. Les énoncés avec $\mathrm{CD}$ en position 1 appellent plusieurs remarques, parce que cette position est soumise à des contraintes différentes :

(30) Adolescent, Mohammed Atta était un garçon solitaire qui ne se joignait pas à ses camarades allant draguer des filles à la sortie du lycée.

(31) ??Enfant, quand on vit dans l'anticipation d'une rupture - parce qu'on en a vécu une traumatisante -, on peut, de façon inconsciente, aller jusqu'à la provoquer.

(32) *Adolescent, entre-temps, il y a eu la pension où il rencontre le peintre Gérard Garouste.

Dans (30), la CD construit le cadre temporel pour le reste du contenu propositionnel, provoquant un changement de portée sémantique assez important. L'exemple (31) relève, de toute évidence, d'un contexte psychologique et, dans l'énoncé initial, on adopte le point de vue d'un adulte pour parler d'une rupture affective, que l'on peut provoquer. Avec la CD[enfant] en position 1, on envisage le reste dans le cadre de l'enfance et, du coup, l'énoncé présuppose non seulement qu'un enfant peut vivre dans l'anticipation d'une rupture, mais qu'il 
a déjà un passé traumatisant et en plus qu'il peut provoquer une telle rupture. Autrement, sans tenir compte d'un tel contexte, l'énoncé sera difficilement acceptable parce que l'on voit mal comment un enfant peut aller jusqu'à provoquer une rupture affective. Enfin, si nous contestons (32), c'est à cause d'un conflit d'intervalles temporels. La CD[adolescent] est immédiatement suivie d'une locution adverbiale entre-temps, qui fait référence à un intervalle temporel borné par deux procès (ou par le début et la fin d'un même procès). Or, si ces deux repères temporels sont supposés être connus (donc figurant dans le contexte à gauche et présents dans la mémoire du lecteur), l'introduction de la CD vient mettre en place l'intervalle temporel les englobant. Généralement, un tel mouvement n'est pas exclu, sauf que, dans notre cas, pour qu'entre-temps puisse référer à l'intervalle temporel « recadré » par la CD, la locution adverbiale doit passer par l'intervalle englobant (instauré par la CD) pour atteindre l'intervalle englobé. Un chemin coûteux qui rend l'énoncé très difficile.

Reprenons les énoncés de départ, (24)-(26), et observons les effets produits par le déplacement de la $\mathrm{CD}$ en position 3 de matrice, immédiatement avant le subordonnant :

??Mohammed Atta était un garçon solitaire, adolescent, qui ne se joignait pas à ses camarades allant draguer des filles à la sortie du lycée.

??Quand on vit dans l'anticipation d'une rupture, enfant - parce qu'on en a vécu une traumatisante -, on peut, de façon inconsciente, aller jusqu'à la provoquer.

*Entre-temps, il y a eu la pension, adolescent, où il rencontre le peintre Gérard Garouste.

En ce qui concerne l'exemple (34), il nous semble être dans le même cas de figure que le déplacement de la $C D$ en tête de phrase. En (33), la position finale de la matrice coïncide avec les compléments attributifs, régis par la copule (être un garçon solitaire). Dans la pratique, les manipulations sur les compléments essentiels du verbe et les circonstants en position finale montrent que la frontière entre les deux est poreuse, surtout quand il s'agit de déterminer l'incidence du circonstant (cf. Serafin 1993). Enfin, le déplacement de la CD en (35) est impossible parce que la matrice ne comporte pas d'élément susceptible de fournir un antécédent. Plus précisément, l'absence de sujet [+humain] dans la matrice, fait que la $\mathrm{CDtmp}(\mathrm{N}$-[âge]) ne peut pas recevoir un support référentiel pour remplir son rôle. Si l'on peut accepter, à la limite un énoncé comme Adolescent, il y a eu la prison, où..., dans lequel on voit que l'iconicité de la position initiale joue un rôle décisif pour l'intelligibilité de l'énoncé ${ }^{22}$, la post-position verbale ne permet pas à la CD d'assurer ce rôle ( ${ }^{*} I l$ y a eu la pension, adolescent, où il...).

22. Même si la $\mathrm{CD}$ [adolescent] co-réfère avec le $i l$ de la subordonnée, on peut très bien imaginer qu'en position initiale elle assure aussi la continuité thématique avec le contexte à gauche, où il est question du parcours de la personne en question. 
Avant de passer à l'étude contrastive avec le bulgare, faisons un bilan rapide des observations faites jusqu'à présent. En plus de dénoter un individu humain, certains NH impliquent une période temporelle d'instanciation et dans des contextes spécifiques (avec des contraintes syntactico-sémantiques, mais aussi d'ordre discursif) peuvent amalgamer plusieurs lectures ${ }^{23}$. Le fait que le prédicat nominal tient à lui seul pour une $\mathrm{CD}$, contribue au caractère interprétatif « flou » de cette configuration et nécessite le recours à d'autres éléments pour son description (phénomènes discursifs, connaissances extra-linguistiques). D'une part, de par leur fonctionnement syntaxique (absence de détermination, prédicats secondaires co-référants), les $\mathrm{CDtmp}(\mathrm{N}$-[âge]) penchent plutôt vers le côté « qualitatif » et se retrouvent dans des gloses attributives (Enfant, il... = il était enfant). D'autre part, de par leur sémantisme propre, les N-[âge] tirent du côté « temporel » ayant le plus souvent pour équivalent sémantique des subordonnées en quand ${ }^{24}$. Nous verrons que, à quelques détails près, le bulgare présente le même fonctionnement ambivalent.

\section{3. ÉTUDE CONTRASTIVE FRANÇAIS-BULGARE}

En bulgare, comme en français, si l'on pense aux CD à valeur circonstancielle, on n'évoque pas d'emblée le domaine nominal ${ }^{25}$. La différence majeure entre le bulgare et le français réside dans le fait que les $\mathrm{N}$-[âge] ne peuvent pas (ou très difficilement) apparaître en emploi « nu » en $\mathrm{CD}^{26}$. Autrement dit, en bulgare, un $\mathrm{N}$-[âge] ne peut pas, à lui tout seul, véhiculer une interprétation temporelle, et une traduction en bulgare avec les contraintes du français sur le $\mathrm{N}$ donnera un énoncé irrecevable :

\section{*Dete, običah spanak.}

Enfant [N. m. sg.], aimais [P1 IMP] épinards [N. m.sg.]

Enfant, j'aimais les épinards.

Pour que l'énoncé soit bien formé, la CD doit être introduite par kato ${ }^{27}$, et remarquons tout de suite que nous n'avons récupéré qu'une partie de la prédication le V n'apparaît pas :

23. Qualitative, temporelle, nous l'avons vu, mais aussi causale : il nous semble que dans Enfant gâté, il faisait tout le temps des caprices, la CD aura plutôt le sens de " parce qu'il était enfant gâté », que de « ?quand il était enfant gâté ».

24. Par exemple, souvent, le contexte impose aux CDtmp(adulte) une lecture résultative (Adulte, il partit faire le tour du monde = Une fois (qu'il est devenu) adulte, il partit faire le tour du monde).

25. Et il semble que ce n'est pas un cas isolé puisque Busuioc fait le même constat pour le roumain : [en roumain] « [...] les participes sont assez fréquents, alors que les adjectifs sont très rares et les noms épithètes ne sont jamais mobilisés dans une CD à valeur circonstancielle. » (Busuioc, 2007 : 234)

26. Les possibilités de position et de mobilité au sein des propositions étant sensiblement les mêmes qu'en français, nous ne développons pas plus en avant ce point. Pour une étude détaillée, cf. Aleksandrova (en cours).

27. À lire [kato], « comme/quand». 
Kato dete, običah spanak.

Quand [conj.sub.] enfant [N m. sg.] aimais [P1 IMP] épinards [N. m.sg.]

Enfant, j'aimais les épinards.

L'étude comparative entre les CD en bulgare et en français montre clairement que la prédication en français peut être réalisée par le seul prédicat nominal ( $N$ [âge] en l'occurrence), contrairement à une réalisation partielle pour le bulgare (conj. + N-[âge]). Il s'en suit que kato $N$-[âge] est une étape intermédiaire vers la réduction maximale qui aura comme réalisation le $\mathrm{N}$-[âge] comme dans (36). Mais quel est le point de départ de cette réduction ? La prédication retrouve sa configuration complète sous la forme d'une subordonnée temporelle, introduite par la conjonction de subordination kogato ${ }^{28}$ (quand en français) :

(38) Kato dete $=$ kogato e bil dete (Quand il était enfant,...)

En d'autres mots, en bulgare, une subordonnée temporelle introduite par la conjonction kogato exige la présence du verbe, tandis que sa réalisation avec kato est partiellement réduite à la conjonction de subordination + le prédicat nominal ${ }^{29}$ :

$$
\text { kogato }+\mathrm{GV}+\mathrm{N} \text {-[âge] } \rightarrow \text { kato }+\mathrm{N} \text {-[âge] }{ }^{30}
$$

Pour le français, la réduction de la prédication est maximale, parce qu'elle se réalise sous une forme minimale, $c^{\prime}$ est-à-dire, le prédicat seul :

$$
\text { Quand + GV + N-[âge] } \rightarrow \text { N-[âge] }{ }^{31}
$$

Terminons ces observations par une remarque importante qui nous permettra de revenir vers l'analyse du français, tout en gardant le fil conducteur de la démonstration. On doit questionner le fait qu'en bulgare, kato peut être soit une préposition (kato $\mathrm{N}$-[âge] = comme enfant), soit une conjonction de subordination comme c'est le cas dans la CDtmp kato $N$-[âge ${ }^{32}$. Le temps verbal de la prédication principale est un paramètre fort de désambiguïsation. Prenons à titre d'exemple les énoncés suivants :

Kato dete e mnogo poslušen. Comme [Prép] enfant [N m.sg.] est [P3 PR] très [Adv] sage [Adj. Attr.] Il est très sage comme enfant.

28. Signalons, qu'en bulgare, kogato peut être une conjonction de subordination (= quand: Quand il était enfant,...) ou bien un adverbe pronominal relatif de temps (= où, Le jour où il est parti,...).

29. À notre connaissance, il n'y a pas de lien entre les deux conjonctions kato/kogato. Des études supplémentaires (notamment en diachronie), qui sortent du cadre de notre travail, devraient apporter plus d'explications sur une éventuelle parenté morphologique, ou bien sur leur distribution respective.

30. Notons qu'en bulgare la subordonnée temporelle ne peut pas avoir la configuration suivante : *Kato (conj.) $+\mathrm{V}+\mathrm{N}$-[âge] (*Comme était enfant,...).

31. Bien évidemment, il ne s'agit que d'une analyse possible.

32. La même question se pose pour comme en français. Parmi les nombreux travaux, cf. Fuchs \& Le Goffic (2005, 2008) ; Léard \& Pierrard (2003); Moline (2005); Moline \& Flaux (2008) ; Pierrard (2002). 
Kato dete beshe mnogo poslušen.

Comme [Conj.Sub.] enfant [N m.sg.] était [P3 IMP] très [Adv.] sage [Adj. Attr.]

Enfant, il était très sage/Quand il était enfant, il était très sage.

À ce propos, notons que l'emploi d'enfant en CD, aussi bien en français qu'en bulgare, impose des contraintes fortes sur le temps verbal de la principale : ${ }^{*}$ Enfant, $j^{\prime}$ aime/j'aimerai le chocolat ${ }^{33}$. La préposition kato (39) ne peut pas être confondue avec la conjonction de subordination (40) parce que le présent dans la principale empêche la circonscription d'une période temporelle passée. Autrement dit, en bulgare, la copule au présent fait que kato $N$-[âge] ne peut porter une charge circonstancielle, et la seule lecture possible est celle dans le cadre du comparatif ${ }^{34}$.

L'étude contrastive avec le bulgare permet une double conclusion. La première concerne la particularité des CD nominales, qui semblent osciller entre des valeurs qualitative et circonstancielle. Nous avons vu que, parmi ces dernières, la valeur temporelle, même si elle est contrainte, n'est pas incompatible avec la nature nominale de la $\mathrm{CD}$. La deuxième est que, si les $\mathrm{CD}$ sont considérées comme des propositions réduites, en bulgare le processus de réduction n'est que partiel, parce que la prédication seconde contenant la CDtmp est introduite par une conjonction de subordination. En revanche, on peut dire que le français est allé jusqu'au bout du processus de réduction parce que le $\mathrm{N}$-[âge] à lui seul peut donner lieu à une valeur temporelle et peut circonscrire une période temporelle pendant laquelle a lieu la prédication principale.

\section{CONCLUSION}

En guise de conclusion, soulignons que ce n'est certainement pas un hasard si les $\mathrm{N}$ se voient attribuer une charge temporelle dans une configuration syntaxique particulière comme les $\mathrm{CD}$. Ce type de construction syntaxique permet une véritable «cohabitation sémantique » entre «qualité » et "temps " ${ }^{35}$. Elle autorise l'accumulation d'un certain nombre de paramètres syntaxico-sémantiques, qui sont à l'origine de son caractère interprétatif «flou ». D'une part, la nonactualisation du N, entraînée par l'absence de déterminant et modifieur fait

\footnotetext{
33. L'espace qui nous est accordé ne nous permet pas de donner une explication plus détaillée à ce fait. Notons qu'au moins deux paramètres entrent en ligne de compte : d'une part, le sémantisme propre de chaque N-[âge] (*enfant, j'aimerai le chocolat vs. adulte, je serai astronaute) et, d'autre part, la relation d'ordre qui régit cet ensemble lexical (on naît bébé, puis on devient enfant, etc.).

34. Notons qu'en le français, le présent peut prendre une valeur dite « historique », qui, tout en rapportant des faits passés, a pour effet de les rendre plus saisissants : Adolescent, il fuit le cercle familial à trois reprises.

35. Cette «dualité » nous semble remise en jeu dans d'autres configurations syntaxiques auxquelles nous envisageons d'étendre l'étude : les N-[âge] en attribut d'objet (cf. Olsson 1976 ; Riegel 1991, 1994, 2001), par ex. Je l'ai connu enfant et les $\mathrm{N}$-[âge] en compléments nominaux : mon pantalon d'adolescent.
} 
de la $C D$ nominale une expression non-référentielle et autorise la valeur circonstancielle. D'autre part, son statut de prédication seconde (extra-prédicative, référentiellement non autonome) permet l'établissement d'une coréférence partielle (tributaire du sémantisme $d u \mathrm{~N}^{36}$ ), qui impose une lecture reflétant la chronologie temporelle (cf. les contraintes sur les temps verbaux). Par ailleurs, et c'est une piste qui mérite d'être approfondie, cette même relation de coréférence, ainsi que l'occupation privilégiée de la position thématique dans une phrase, font que les $\mathrm{CD}(\mathrm{N}$-[âge]) participent dans la construction textuelle sur deux plans : ancrage et balisage temporel de la narration d'une part, maintient thématique et continuité référentielle, de l'autre.

En somme, on peut dire que, si la réalisation ${ }^{37}$ d'une prédication dans un constituant nominal entraîne inévitablement la perte de ses capacités expressives de prédication complète, le $\mathrm{N}$ est à même d'assumer des interprétations diverses. Dans une perspective comparative, les résultats ont montré que, même si les $\mathrm{NH}$, aussi bien en français qu'en bulgare, peuvent véhiculer des sens identiques (tantôt qualificatifs, tantôt temporels), leur emploi en $C D$ varie en fonction des contraintes spécifiques : de détermination pour le français, de rapport avec le GV pour le bulgare. Enfin, en guise de conclusion, notons qu'une future étude devrait prendre en compte l'examen d'autres configurations susceptibles de véhiculer une valeur temporelle (comme enfant ${ }^{38}$ ), qui montrera éventuellement l'existence du paradigme complet pour le français (Quand j'étais enfant/Comme enfant/Enfant, je préférais passer mes vacances au bord de la mer). L'intérêt est d'autant plus grand que, dans une perspective contrastive, dans de nombreuses langues l'introduction du N-[âge] est obligatoire : as a child (eng.), als Kind (all.), come un bambino (it.), como un niño (esp.), ca un copil ${ }^{39}$ (roum.).

\section{Références}

CORPUS BRAUn (BULGARE) - http://dcl.bas.bg/Corpus/publications_bg.html

CORPUS NATIONAL BULGARE - http://www.ibl.bas.bg/BGNC_bg.htm

FRANTEXT (FRANÇAIS) - http://www.frantext.fr/

WORTSCHATZ (FRANÇAIS) - http://wortschatz.uni-leipzig.de/ws_fra/

36. Il faut que le $\mathrm{N}$ soit un $\mathrm{N}$ de phase : *(Quand il était) Albinos, il restait à l'écart.

37. Dans son travail sur la composition nominale, Benveniste observe le même phénomène (l'expression d'une prédication dans un « format » nominal) et y voit une forme particulière du processus plus général de «transformation de certaines classes en autres », qu'il propose d'appeler « métamorphisme » (Benveniste, 1974 : 161).

38. Nous tenons à remercier Dejan Stosic pour cette suggestion.

39. En roumain, la préposition $c a$ (« comme ») est évitée pour des raisons de cacophonie $c a-c o$ [ka-ko] dans *Ca copil (« comme enfant »), mais elle peut apparaître dans ce type de structures : Ca student, citeam Balzac (Comme étudiant, je lisais Balzac). L'insertion d'un déterminant, ca un copil, fait basculer le sens dans le comparatif. Nous remercions M. Navlea pour les précisions sur ces subtilités d'emploi. 
Aleksandrova A. (2010), "Prédicats d'état et temporalité : l'exemple des prédicats d'âge ", Interlinguistica XXI, 805-815.

Aleksandrova A. (en cours), Problèmes de classifications ontologiques et linguistiques: l'exemple des noms d'humains de phase, Thèse de l'Université de Strasbourg.

AMOURETTE C. (2004), L'expression du temps et de l'aspect dans les formes non conjuguées du français, Thèse de I'Université de Rouen.

AMOURETTE C. (2009), "Sémantique temporelle de quelques constructions appositives ", in D. Apothéloz, B. Combettes \& F. Neveu (éds), Les Linguistiques du détachement, Berne : Peter Lang, 33-49.

Anscombre J.-C. (1990), "Article zéro et structuration d'événements ", in M. Charolles, S. Fisher \& J. Jayez (éds), Le discours : représentations et interprétations, Nancy : Presses Universitaires de Nancy, 265-295.

Apothéloz D., Combettes B. \& Neveu F. (éds) (2009), Les Linguistiques du détachement, Berne : Peter Lang.

Benveniste É. (1974), Problèmes de linguistique générale, vol. 2, Paris : Gallimard.

BLANCHE-BENVENISTE C. (1998), "L'usage prédicatif secondaire des participe passés ", in M. Forsgren, K. Jonasson \& H. Kronning (éds), Prédication, assertion, information, Uppsala : Acta Universitatis Uppsaliensis, 43-56.

Borillo A. (2006), "Quelques structures participales de valeur temporelle en prédication seconde ", Travaux Linguistiques du CERLICO 19, 167-182.

BoriLlo A. (2009), " Le participe passé comme mode d'expression d'une prédication seconde de sens temporel ", in D. Apothéloz, B. Combettes \& F. Neveu (éds), Les Linguistiques du détachement, Berne : Peter Lang, 129-151.

Busuioc I. (2007), " Détachée, cette construction serait ambiguë. Valeurs circonstancielles des modificateurs du nom détachés en français et en roumain ", in N. Flaux \& D. Stosic (éds), Les constructions détachées : entre langue et discours, Arras : Artois Presses Université, 229-240.

Combettes B. (1998a), Les constructions détachées en français, Paris : Ophrys.

ComBetTes B. (1998b), "Prédication et perspective fonctionnelle de la phrase : le cas des constructions détachées ", in M. Forsgren, K. Jonasson \& H. Kronning (éds), Prédication, assertion, information, Uppsala : Acta Universitatis Uppsaliensis, 129-138.

Combettes B. (2005), "Les constructions détachées comme cadres de discours ", Langue française 148, 31-44.

FAuCONNIER G. (1984), Espaces mentaux, Paris : Editions de Minuit.

Flaux N. \& Stosic D. (éds) (2007), Les constructions détachées : entre langue et discours, Arras : Artois Presses Université.

ForsGREN M. (2005), "Apposition, attribut, épithète : même combat prédicatif ? ", Langue française 125, 30-45.

Forsgren M., Jonasson K. \& Kronning H. (éds) (1998), (éds), Prédication, assertion, information, Uppsala : Acta Universitatis Uppsaliensis.

Fuchs C. \& FOURNIER N. (2003), " Du rôle cadratif des compléments localisants initiaux selon la position du sujet nominal ", Travaux de linguistique 47, 79-109.

Fuchs C. \& LE Goffic P. (2005), "La polysémie de comme ", in O. Soutet (éd.), La polysémie, Paris : Presses Universitaires Paris-Sorbonne, 267-291.

Fuchs C. \& LE GofFIC P. (2008), "Un emploi typifiant de comme: un de ces exemples comme on en trouve partout ", Langue française 159, 67-82. 
HAVU E. (2002a), " Sur quels principes l'interprétation des constructions détachées repose-telle ?", Romansk forum 16, Actes du XVe Congrès des romanistes scandinaves, 389-400. [www.duo.uio.no/roman/page21.html]

Havu E. (2002b), "L'interprétation des constructions détachées ", CLAC 10. [www.ucm.es/info/circulo/no10/index.htm]

Havu E. \& Pierrard M. (2007), " Détachement et prédication seconde ", Neuphilologische Mitteilungen 4, 729-742.

LÉARD J.-M. \& PierRaRd M. (2003), "L'analyse de comme: le centre et la périphérie ", in P. Hadermann, A. Van Sijcke \& M. Berré (éds), La syntaxe raisonnée, Bruxelles : Duculot, 203-234.

Le QueRLeR N. (1993), "Les circonstants et la position initiale ", in C. Guimier (éd.), 1001 Circonstants, Caen : Presses Universitaires de Caen, 159-185.

Moline E. (2005), "Nous venons ici comme témoins. À propos des emplois qualifiants de comme ", in M. Tenchéa \& A. Tihu (éds), Prépositions et conjonctions de subordination. Syntaxe et sémantique, Timisoara : Excelsior Art Editura, 159-174.

Moline E. \& Flaux N. (2008), "Constructions en comme : homonymie ou polysémie ? Un état de la question ", Langue française 159, 3-9.

OLSSON K. (1976), La construction verbe+objet direct+complément prédicatif en français. Aspects syntaxiques et sémantiques, Stockholm : Göteborgs Offsettryckeri.

PICABIA L. (2000), "Appositions nominales: les cas des appositions frontales ", Langue française 125, 71-89.

Pierrard M. (2002), "Comme préposition ? Observations sur le statut catégoriel des prépositions et des conjonctions ", Travaux de linguistique 44, 69-78.

RIEGEL M. (1985), L'adjectif attribut, Paris : Presses Universitaires de France.

RIEGEL M. (1991), "Pour ou contre la notion grammaticale d'attribut de l'objet : critères et arguments ", in M.-M. De Gaulmyn \& S. Rémi-Giraud (éds), À la recherche de l'attribut, Lyon : Presses Universitaires de Lyon, 99-119.

RIEGEL M. (1994), "La catégorie grammaticale de l'attribut ", Le Gré des langues 7, 170-189.

RIEGEL M. (2001), "Quelques remarques sur les constructions à l'attribut de l'objet issues d'une complétive attributive ", in $\mathrm{H}$. Kronning et al. (éds), Langage et référence, Uppsala : Acta Universitatis Upsaliensis, 543-555.

SERAFIN V. (1993), "Les circonstants en position finale : problèmes d'incidence ", in C. Guimier (éd.), 1001 Circonstants, Caen : Presses Universitaires de Caen, 207-235.

SWART H. (DE), WINTER Y. \& ZWARTS J. (2007), "Bare nominals and reference to capacities", Natural language \& Linguistics Theory 25, 195-222.

WIGGINS D. (2001), Sameness and substance renewed, Cambridge: Cambridge University Press.

\section{Liste des abréviations}

$C D$ : construction détachées

CDqua : construction détachée à valeur qualitative ou descriptive

CDtmp : construction détachée à valeur circonstancielle de temps

$\mathrm{CD}(\mathrm{X})$ : X - type de NH en CD (par ex. CD (Npro) : un nom de profession en construction détachée)

$\mathrm{NH}$ : noms d'humains (référents humains) 